\title{
Guía de práctica clínica para el tamizaje, diagnóstico y manejo de la enfermedad renal crónica en estadios 1 al 3 en el Seguro Social del Perú (EsSalud)
}

\section{Clinical practice guideline for the screening, diagnosis, and management of chronic kidney disease stages 1-3 for the Peruvian Social Security (EsSalud)}

Jessica Bravo-Zúñiga 1, 2,a,b,c, Juana Hinostoza-Sayas ${ }^{3, a, b}$, Sergio GoicocheaLugo 2,a, Gandy Dolores-Maldonado 2,d,e, Ana Brañez-Condorena ${ }^{4, f}$, Alvaro TaypeRondan 2,a, , Carlos Yanar Pereda Vejarano ${ }^{3, a, b}$, Carola Medina Sal y Rosas ${ }^{1, a, b}$, Renzo P. Valdivia-Vega ${ }^{1, a, b}$, Fernando Mesías Gonzáles Haro ${ }^{5, a, b}$, Luzmila Livaque Gaona ${ }^{6, a}$, Victor Manuel Conetero Muro7,a, Gabriela Ayllón Guerrero 8,a,h, Nelly Patricia Huancco Cáceres ${ }^{9, a, h}$, César Antonio Loza Munarriz ${ }^{10, a, i}$, Percy Herrera-Añazco 11,a,b,j, Virgilio E. Failoc Rojas ${ }^{2, a}$, Lourdes Carrera-Acosta ${ }^{2, a, k}$, Raúl Timaná Ruiz $z^{2, a, k, l}$, Héctor Garavito-Farro ${ }^{2, a, m}$

Hospital Nacional Edgardo Rebagliati Martins, EsSalud. Lima, Perú.

Instituto de Evaluación de Tecnologías en Salud e Investigación, EsSalud. Lima, Perú.

Centro Nacional de Salud Renal, EsSalud. Lima, Perú.

Universidad Nacional Mayor de San Marcos, Facultad de Medicina. Lima, Perú.

Hospital Nacional Alberto Sabogal Sologuren, EsSalud. Lima, Perú.

Hospital III de Emergencias Grau, EsSalud. Lima, Perú.

Centro de Atención Primaria (CAP) III Luis Negreiros Vega, EsSalud. Lima, Perú

Policlínico Chincha, EsSalud. Lima, Perú.

Hospital Vitarte, MINSA. Lima, Perú

Hospital Cayetano Heredia, MINSA. Lima, Perú.

Universidad Peruana de Ciencias Aplicadas. Lima, Perú

Médico cirujano; ${ }^{b}$ especialista en Nefrología; ${ }^{\mathrm{C}}$ maestro en Epidemiología clínica; ${ }^{\mathrm{d}}$ licenciada en Nutrición; ${ }^{\mathrm{e}}$ maestro en Gerencia social con mención en desarrollo económico local; ${ }^{f}$ estudiante de Medicina; ${ }^{8}$ maestro en Ciencias en investigación epidemiológica; ${ }^{\mathrm{h}}$ especialista en Medicina familiar; ' maestría en Medicina; ${ }^{\mathrm{j}}$ maestro en Docencia en educación superior; ${ }^{\mathrm{k}}$ especialista en Gestión en salud; ' maestro en Farmacoeconomía / Economía de la salud y del medicamento; ${ }^{m}$ maestro en Ciencias: Salud comunitaria en países en desarrollo.

Correspondencia Héctor Garavito-Farro hector.garavito@essalud.gob.pe

Recibido: 23/09/2020 Arbitrado por pares Aprobado: $12 / 11 / 2020$

Citar como: Bravo-Zúñiga J, Hinostoza-Sayas J, Goicochea-Lugo S, Dolores-Maldonado G, BrañezCondorena A, Taype-Rondan Á, et al. Guía de práctica clínica para el tamizaje, diagnóstico y manejo de la enfermedad renal crónica en estadios 1 al 3 en el Seguro Social del Perú (EsSalud). Acta Med Peru. 2020;37(4):518-31. doi: https://doi. org/10.35663/amp.2020.374.1843

\section{RESUMEN}

El presente artículo resume la guía de práctica clínica (GPC) para tamizaje, diagnóstico y manejo de los pacientes con enfermedad renal crónica (ERC) en los estadios 1 al 3 en el Seguro Social del Perú (EsSalud). Para el desarrollo de esta GPC, se conformó un grupo elaborador de la guía (GEG) que incluyó especialistas clínicos y metodólogos, el cual formuló ocho preguntas clínicas. Para responder cada pregunta se realizaron búsquedas sistemáticas de revisiones sistemáticas y, cuando fue considerado pertinente, de estudios primarios; $y$ se seleccionó la evidencia pertinente. La certeza de la evidencia fue evaluada usando la metodología Grading of Recommendations Assessment, Development, and Evaluation (GRADE). En reuniones periódicas, el GEG usó la metodología GRADE para revisar la evidencia y emitir las recomendaciones. Se emitieron ocho recomendaciones (cuatro fuertes y cuatro condicionales), 29 puntos de buena práctica clínica, y tres flujogramas.

Palabras clave: Insuficiencia renal crónica; Guía de práctica clínica; Enfoque GRADE; Medicina basada en la evidencia (fuente: DeCS BIREME). 


\section{ABSTRACT}

This paper summarizes the clinical practice guidelines (CPG) for the screening, diagnosis, and management of patients with chronic kidney disease (CKD) stages 1-3 in the Social Security of Peru (EsSalud). A guideline development group (GDG) was established for develop this CPG, which included clinical and methodology specialists, who formulated 08 clinical questions. Systematic searches of systematic reviews and, when considered necessary, primary studies were conducted to answer each question; and relevant evidence was selected. The certainty of the evidence was assessed using the Grading of Recommendations Assessment, Development, and Evaluation (GRADE) methodology. In periodic work meetings, the GDG used the GRADE methodology for reviewing the evidence and for developing recommendations. At the end, this CPG formulated 08 recommendations ( 04 strong and 04 conditional), 29 points of good clinical practice, and 03 flowcharts were formulated.

Keywords: Chronic renal insufficiency, Practice guidelines, GRADE approach, Evidence-based medicine (source: MeSH NLM).

\section{INTRODUCCIÓN}

La enfermedad renal crónica (ERC) consiste en una pérdida progresiva de la función renal a través de cinco estadios ${ }^{[1,2]}$. Se estima que la ERC afecta del $8 \%$ a $16 \%$ de la población y tanto la incidencia como mortalidad van en aumento a nivel mundial ${ }^{[3]}$. En el Perú, un estudio realizado en Lima y Tumbes reportó que la prevalencia de ERC fue de $20,7 \%$ y $12,9 \%$, respectivamente, en el año $2011^{[4]}$.

La tendencia creciente tanto en la incidencia como en la mortalidad de la ERC, dan cuenta que a pesar de contar con estrategias terapéuticas para su manejo, los pacientes son captados en estadios avanzados ${ }^{[5]}$. Ante ello, se ha propuesto que la evaluación y el manejo oportuno y adecuado de los casos de ERC, principalmente en estadios tempranos ( 1 al 3), reducirían la morbimortalidad y las complicaciones de esta condición, evitando que esta enfermedad impacte en la calidad de vida de las personas que la padecen ${ }^{[1,5,6]}$. En consecuencia, el Seguro Social de Salud (EsSalud) priorizó la elaboración de la presente guía de práctica clínica (GPC) para establecer lineamientos basados en evidencia y gestionar de la mejor manera los procesos y procedimientos asistenciales de la presente condición.

Por ello, la Dirección de Guías de Práctica Clínica, Farmacovigilancia y Tecnovigilancia del Instituto de Evaluación de Tecnologías en Salud e Investigación (IETSI) del Seguro Social del Perú (EsSalud) elaboró la guía de práctica clínica (GPC) basada en evidencias para el tamizaje, diagnóstico y manejo de la ERC en estadios 1 al 3, cuyas recomendaciones (Tabla 1) serán aplicadas por profesionales de la salud en EsSalud. El presente artículo es un resumen de dicha GPC.

\section{METODOLOGÍA}

El procedimiento seguido para la elaboración de la presente GPC está detallado en su versión completa, la cual puede descargarse de la página web del IETSI de EsSalud (http://www.essalud. gob.pe/ietsi/guias_pract_clini.html). En resumen, se aplicó la siguiente metodología:
Conformación del grupo elaborador de la guía (GEG): Se conformó un GEG, que incluyó expertos en la metodología de la elaboración de GPC y médicos especialistas clínicos.

Formulación de preguntas: En concordancia con los objetivos y alcances de esta GPC, el GEG formuló 8 preguntas clínicas (Tabla 1), cada una de las cuales pudo tener una o más preguntas PICO (Population, Intervention, Comparator, Outcome).

Búsqueda y selección de la evidencia: Entre 2018 y 2019, para cada pregunta PICO se buscaron revisiones sistemáticas (RS) publicadas como artículos científicos (mediante búsquedas sistemáticas en PubMed) o realizadas como parte de una GPC previa (mediante una búsqueda sistemática de GPC sobre el tema) (Material suplementario 1). Cuando se encontraron RS de calidad aceptable, se escogió una RS para la toma de decisiones en base al puntaje obtenido con el instrumento $A$ MeaSurement Tool to Assess systematic Reviews (AMSTAR-2), la fecha en la que realizó la búsqueda sistemática, y el número de estudios incluidos. Dicha RS fue actualizada cuando el GEG lo consideró necesario (mediante búsquedas sistemáticas en PubMed). Cuando no se encontró ninguna RS de calidad aceptable, se realizó una búsqueda de novo de estudios primarios (mediante búsquedas sistemáticas en PubMed, Scopus, y/o Biblioteca Regional de Medicina [BIREME]).

Evaluación de la certeza de la evidencia: La certeza de la evidencia para cada desenlace de cada pregunta PICO pudo ser alta, moderada, baja, o muy baja (Tabla 2). Para evaluar la certeza de la evidencia, se siguió la metodología de Grading of Recommendations Assessment, Development, and Evaluation $(\text { GRADE })^{[7]}$ y se usaron tablas de perfiles de evidencias de GRADE (Material suplementario 2). Finalmente, se le asignó a la pregunta PICO el nivel de certeza más bajo alcanzado por alguno de los desenlaces críticos.

Formulación de las recomendaciones: El GEG revisó la evidencia seleccionada para cada pregunta clínica en reuniones periódicas, y formuló recomendaciones fuertes o condicionales (Tabla 2) usando la metodología GRADE [8]. Para ello, se tuvo en consideración: 1) Beneficios y daños de las opciones, 2) Valores y preferencias de los pacientes, 3) Aceptabilidad por parte de los profesionales de salud, 4) Factibilidad de las opciones en 
Tabla 1. Lista completa de recomendaciones.

Tamizaje, diagnóstico y estadiaje de ERC

Pregunta 1: En adultos, ¿se debería realizar el tamizaje de enfermedad renal crónica durante la atención primaria?

En adultos, recomendamos realizar el tamizaje de enfermedad renal crónica dirigido a grupos de riesgo.

En adultos, considerar como grupos de riesgo a: pacientes con diabetes mellitus tipo 102 , hipertensión arterial, o mayores de 55 años.

En adultos, considerar realizar el tamizaje a personas con otros factores de riesgo para ERC según criterio clínico. Entre los factores a considerar se encuentran:

- Antecedente de injuria renal aguda (IRA)

- Enfermedades cardiovasculares

- Enfermedades que alteren la estructura renal o el tracto urinario

- Enfermedades multisistémicas con potencial daño renal

- Historia familiar de falla renal

- Presencia de hematuria

- Escenarios laborales con potencial daño renal (por ejemplo, exposición a pesticidas, deshidratación)

En adultos, utilizar el cálculo de la tasa de filtración glomerular estimada (TFGe) junto con la determinación de albuminuria mediante la relación albúminuria-creatinuria (RAC) para realizar el tamizaje de ERC [Ver pregunta 2 y 3]. Se definirá tamizaje positivo cuando se obtenga una TFGe < $60 \mathrm{ml} / \mathrm{min} / 1,73 \mathrm{~m}^{2} \mathrm{y} / \mathrm{o}$ RAC $\geq 30 \mathrm{mg} / \mathrm{g}$.

En adultos a quienes se realice el tamizaje de ERC y se obtenga una TFGe $<60 \mathrm{ml} / \mathrm{min} / 1,73 \mathrm{~m}^{2}$ con o sin criterios clínicos de IRA, realizar una nueva medición de la TFGe dentro de las siguientes dos semanas para confirmar el diagnóstico. En caso se confirme el diagnóstico de IRA, referir al especialista en nefrología.

En adultos con un primer tamizaje positivo para ERC en quienes se haya descartado IRA, repetir el tamizaje luego de tres meses para confirmar el diagnóstico de ERC. El diagnóstico de ERC se confirmará cuando el paciente presente alguna de las siguientes condiciones:

- TFGe $\geq 60 \mathrm{ml} / \mathrm{min} / 1,73 \mathrm{~m}^{2}$ y albuminuria (RAC $\geq 30 \mathrm{mg} / \mathrm{g}$ ) ó

- TFGe $<60 \mathrm{ml} / \mathrm{min} / 1,73 \mathrm{~m}^{2}$

En adultos con un primer tamizaje negativo para ERC, considerar repetir el tamizaje tomando en cuenta el valor de TFGe obtenido, los factores de riesgo del paciente y criterio del médico tratante. En caso se concluya que el tamizaje es negativo, repetir el tamizaje anualmente.

En adultos con diagnóstico de ERC, realizar la clasificación del estadio de la enfermedad según los criterios del grupo de trabajo de enfermedad renal crónica publicados en 2012 (Kidney Disease: Improving Global Outcomes, KDIGO - 2012).

Clasificación del estadio de Enfermedad Renal Crónica (ERC) según KDIGO (Kidney Disease: Improving Global Outcomes)

\begin{tabular}{|c|c|c|c|c|}
\hline & & \multicolumn{3}{|c|}{ Categorías de albuminuria (mg/g) † } \\
\hline Estadio & & $\begin{array}{l}\text { A1 (<30): } \\
\text { Norm }\end{array}$ & $\begin{array}{c}\text { A2 (30-300): } \\
\text { Aumento } \\
\text { moderado }\end{array}$ & $\begin{array}{c}\text { A3 (>300): Aumento } \\
\text { severo }\end{array}$ \\
\hline \multirow{6}{*}{$\begin{array}{l}\text { Categorías de } \\
\text { TFGe } *(\mathrm{ml} / \\
\left.\min / 1,73 \mathrm{~m}^{2}\right)\end{array}$} & G1 ( $\geq 90)$ : Normal o alto & \multirow{2}{*}{ No ERC } & & \\
\hline & G2 (60-89): Reducción leve & & & \\
\hline & $\begin{array}{c}\text { G3a (45-59): Reducción } \\
\text { leve-moderada }\end{array}$ & & & \\
\hline & $\begin{array}{l}\text { G3b (30-44): Reducción } \\
\text { moderada-severa }\end{array}$ & & & \\
\hline & $\begin{array}{c}\text { G4 (15-29): Reducción } \\
\text { severa }\end{array}$ & & & \\
\hline & G5 (<15): Falla renal & & & \\
\hline $\begin{array}{l}\text { * TFGe: Tasa de filtr } \\
+ \text { Albuminuria obte }\end{array}$ & $\begin{array}{l}\text { ón glomerular estimada, calculada } \\
\text { la mediante la relación albuminuri }\end{array}$ & ediante la ecua & $\begin{array}{l}\text { n CKD-EPI creatin } \\
\text { ) [Ver pregunta } 3\end{array}$ & a [Ver pregunta 2]. \\
\hline$\square$ Riesgo leve d & $\square$ Riesgo mode & do de progresic & Riesg & severo de progresión \\
\hline
\end{tabular}

Recomendación Muy baja a favor fuerte $(\bigoplus \Theta \Theta \Theta)$

BPC

BPC

BPC

BPC

BPC

BPC 
Tabla 1. Lista completa de recomendaciones. (viene de la pág. 520)

Tamizaje, diagnóstico y estadiaje de ERC

Pregunta 2: En adultos, ¿qué ecuación se debería utilizar para calcular la tasa de filtración glomerular estimada: ecuación Colaboración Epidemiológica para Enfermedad Renal Crónica (CKD-EPI) o la ecuación de Modificación de la Dieta en Enfermedad Renal con dilución isotópica con espectrometría de masa (MDRD4)?

En adultos, sugerimos utilizar la ecuación CKD-EPI creatinina para el cálculo de la tasa de filtración Recomendación a Muy baja glomerular estimada (TFGe). favor condicional $(\oplus \Theta \Theta \Theta)$

En adultos en quienes se realice el cálculo de la TFGe, utilizar métodos enzimáticos para la determinación de creatinina que sean equiparables a la dilución isotópica y espectrometría de masas (Isotope Dilution Mass Spectrometry, IDMS).

Cuando se solicite la evaluación de la función renal como examen auxiliar, el laboratorio debe reportar el valor de la TFGe en mililitros por minuto por 1,73 metros cuadrados ( $\mathrm{ml} / \mathrm{min} / 1,73 \mathrm{~m}^{2}$ ) y el valor de la creatinina sérica en las unidades miligramos por decilitro $(\mathrm{mg} / \mathrm{dl})$.

BPC

BPC

Pregunta 3: En adultos, ¿qué método se debería utilizar para la detección de albuminuria: relación albuminuria-creatinuria (RAC), relación proteinuria-creatinuria (RPC) o tiras reactivas en orina?

En adultos, recomendamos utilizar la relación albuminuria-creatinuria (RAC) para la determinación de albuminuria.

Recomendación a favor fuerte

Ваја

En adultos, considerar no utilizar el tipo de tira reactiva para examen rutinario de orina con el que cuenta el seguro social con el objetivo de determinar albuminuria. En lugar de ello, considerar su uso para descartar infección urinaria previo a la determinación de RAC en caso exista sospecha de infección.

Cuando no se encuentre disponible la RAC en el centro de salud al que acuda inicialmente al paciente o en un laboratorio de referencia del seguro social, considerar el uso de la relación proteinuriacreatinuria (RPC) teniendo precaución en la interpretación de los resultados.

En adultos en quienes se requiera una mayor precisión para determinar los niveles de albuminuria, considere medir la excreción urinaria de albúmina en una muestra de orina recolectada durante 24 horas como complemento de la RAC o RPC.

En adultos en quienes se realice la detección de albuminuria con RAC o RPC, obtener estos valores a partir de una muestra que contenga la primera orina de la mañana o de una muestra de orina al azar, habiendo descartado el primer chorro de micción en ambos casos.

Cuando se solicite la medición de albuminuria como examen auxiliar, el laboratorio debe reportar el valor de la RAC en miligramos de albúmina por gramo de creatinina $(\mathrm{mg} / \mathrm{g}$ ).
BPC

BPC

BPC

BPC

BPC

\section{Manejo no farmacológico de la ERC}

Pregunta 4: En adultos con enfermedad renal crónica en estadio 1 al 3, ¿qué tipo de dieta se debería brindar: dieta baja en proteínas, muy baja en proteínas o normoproteica?

\section{Pacientes con ERC sin diabetes mellitus}

En adultos con ERC en estadio 1 al 3 sin diabetes mellitus, sugerimos no ofrecer dieta baja en Recomendación proteínas (DBP) para evitar la progresión de la ERC.

en contra

condicional

En adultos con ERC en estadio 1 al 3 sin diabetes mellitus, recomendamos no ofrecer dieta muy baja en proteínas (DMBP) para evitar la progresión de la ERC.

Recomendación Muy baja en contra fuerte $(\bigoplus \Theta \Theta \Theta)$

Respecto al uso de dietas con restricción en la cantidad de proteínas, considerar definir como DBP cuando se brinde $\leq 0,6$ a 0,8 g/kg/día, y DMBP cuando se brinde $<0,4 \mathrm{~g} / \mathrm{kg} /$ día.

BPC

(Continúa en la pág. 522) 
Tabla 1. Lista completa de recomendaciones. (viene de la pág. 521)

\begin{tabular}{lll} 
& Enunciado & Tipo * Certeza ** \\
\hline Tamizaje, diagnóstico y estadiaje de ERC & & \\
\hline
\end{tabular}

En adultos con ERC en estadio 1 al 3, considerar la interconsulta a profesionales de nutrición para realizar la valoración nutricional inicial, establecer un régimen dietario personalizado en el cual se consideren factores socioculturales, preferencias de alimentación del paciente y establecer la frecuencia de monitoreo del estado nutricional.

En adultos con ERC en estadio 1 al 3, considerar establecer el consumo adecuado de calorías, proteínas, y sal de manera individualizada como parte de la formulación del régimen dietario, evitando ofrecer más de la cantidad de proteínas recomendada como normal y una dieta alta en sal.

En adultos con ERC en estadio 1 al 3, aconsejar seguir un estilo de vida saludable que incluya el mantenimiento de peso adecuado, realización de ejercicio físico, evitar hábitos nocivos, y seguir un régimen dietario adecuado como parte integral del manejo de enfermedades crónicas.

BPC

BPC

$\mathrm{BPC}$

\section{Pacientes con ERC sin diabetes mellitus}

En adultos con ERC en estadio 1 al 3 y diabetes mellitus tipo 1 o 2, sugerimos no brindar dieta baja en proteínas (DBP) para evitar la progresión de la ERC.

Recomendación

en contra condicional
Muy baja $(\oplus \Theta \Theta \Theta)$

\section{Manejo farmacológico de la ERC}

Pregunta 5: En adultos con enfermedad renal crónica en estadio 1 al 3 e hipertensión arterial, ¿̇e debería brindar inhibidores de la enzima convertidora de angiotensina (IECA) o antagonistas del receptor de angiotensina-II (ARA-II) para prevenir la progresión de la enfermedad renal crónica?

En adultos con ERC en estadio 1 al 3 con hipertensión arterial y/o diabetes mellitus que cursen con albuminuria, recomendamos brindar IECA o ARA-II para evitar la progresión de la ERC.

Recomendación Moderada

En adultos con ERC en estadio 1 al 3 e hipertensión arterial, considerar incluir el uso de IECA o ARA-II como primera opción de esquema terapéutico antihipertensivo dados los beneficios adicionales en la prevención de progresión de la ERC.

En adultos con ERC en estadio 1 al 3 normotensos, considerar realizar un examen clínico integral con el fin de confirmar o descartar hipertensión arterial que no haya sido diagnosticada previamente. En caso se descarte hipertensión arterial, considerar brindar IECA o ARA-II cuando se detecte albuminuria (A2 - A3).

En adultos con ERC en estadio 1 al 3, considerar las preferencias del paciente, contraindicaciones al uso de IECA y/o presencia de tos luego del uso de IECA, para decidir iniciar o realizar el cambio de la terapia por ARA-II.

En adultos con ERC en estadio 1 al 3, evitar el uso combinado de IECA y ARA-II para prevenir la progresión de ERC dado que su uso incrementa del riesgo de eventos adversos (tos, hipercalemia, hipotensión, IRA que requiere diálisis) sin ofrecer mayores beneficios en comparación con la monoterapia.

a favor fuerte $(\bigoplus \bigoplus \oplus \Theta)$

Pregunta 6: En adultos con enfermedad renal crónica en estadio 1 al 3 y dislipidemia, ¿se debería brindar estatinas para prevenir la progresión de la enfermedad renal crónica?

En adultos con ERC en estadio 1 al 3 y dislipidemia, sugerimos no brindar estatinas solo para prevenir la progresión de la ERC.

Recomendación
en contra
condicional $\quad(\bigoplus \oplus \Theta \Theta)$

En adultos con ERC en estadio 1 al 3 y dislipidemia, mantener el tratamiento con estatinas como parte del manejo de la dislipidemia cuando haya sido indicado.

BPC

En adultos con ERC en estadio 1 al 3 y dislipidemia en quienes se haya presentado eventos cardiovasculares y/o cerebrovasculares, mantener el tratamiento preventivo de estos eventos con estatinas cuando haya sido indicado.

$\mathrm{BPC}$

$\mathrm{BPC}$

$\mathrm{BPC}$

BPC 
Tabla 1. Lista completa de recomendaciones. (viene de la pág. 522)

\begin{tabular}{|lcc} 
& Enunciado & Tipo * Certeza ** \\
\hline Tamizaje, diagnóstico y estadiaje de ERC & \\
\hline Monitoreo de los pacientes con ERC
\end{tabular}

Pregunta 7: En adultos con enfermedad renal crónica en estadio 1 al 3, ¿cuál debe ser la frecuencia de monitoreo de la tasa de filtración glomerular estimada (TFGe) y albuminuria para evaluar la progresión y el estadio de la enfermedad renal crónica?

No se emitieron recomendaciones para esta pregunta.

En adultos con ERC en estadios 1 al 2, considerar monitorear el cambio en la TFGe y albuminuria una vez al año.

BPC

BPC

En adultos con ERC en estadio 3 o con albuminuria $\geq 300 \mathrm{mg} / \mathrm{g}$ (RAC o equivalente), considerar monitorear el cambio en la TFGe y albuminuria dos veces al año.

En adultos con ERC en estadio 1 al 3, queda a criterio clínico realizar un menor o mayor número de controles de TFGe y albuminuria teniendo en cuenta factores de riesgo de progresión del paciente (hipertensión arterial o diabetes mellitus con mal control, cambio en el rango de albuminuria, disminución rápida de la TFGe o nivel de TFGe).

En adultos con ERC, monitorear la presencia de complicaciones de ERC tales como hipercalemia, acidosis metabólica, hiperfosfatemia, deficiencia de vitamina $D$, hiperparatiroidismo secundario, anemia, o alteraciones del metabolismo mineral óseo según el estadio de la enfermedad.

\section{Criterios de referencia}

Pregunta 8: En adultos con enfermedad renal crónica en estadio 1 al 3, ¿cuáles son los criterios de referencia al especialista en nefrología?

No se emitieron recomendaciones para esta pregunta.

En adultos con ERC en estadio 1 al 3, considerar los siguientes criterios para referir a especialista en nefrología:

- Pacientes con TFGe $<30 \mathrm{~mL} / \mathrm{min} / 1,73 \mathrm{~m}^{2}$.

- Pacientes con TFGe $\geq 30$ y $<45 \mathrm{~mL} / \mathrm{min} / 1,73 \mathrm{~m}^{2}$ y albuminuria $>300 \mathrm{mg} / \mathrm{g}$ (RAC o equivalente).

- Pacientes con deterioro agudo de la función renal (descenso de la TFGe $>25 \%$ en un mes respecto a la TFGe basal o disminución mayor de $5 \mathrm{ml} / \mathrm{min} / 1,73 \mathrm{~m}^{2}$ en un año).

- Pacientes con albuminuria $>300 \mathrm{mg} / \mathrm{g}$ (RAC o equivalente) y hematuria de etiología desconocida.

- Pacientes con ERC e injuria renal aguda.

- Pacientes hipertensos con inadecuado control de la presión arterial (presión arterial mayor de $130 / 80 \mathrm{mmHg}$ ) a pesar del uso de tres fármacos antihipertensivos a dosis plenas y al menos un diurético.

- Pacientes con ERC que presenten anemia normocítica normocrómica (hemoglobina $<10,5 \mathrm{~g} / \mathrm{dL}$ ) luego de haber corregido la ferropenia y habiendo descartado causas no renales de anemia.

- Pacientes con alteraciones persistentes en el potasio $(>5,5 \mathrm{mEq} / \mathrm{L} 0<3,5 \mathrm{mEq} / \mathrm{L})$ en quienes no se haya brindado tratamiento con diuréticos.

- Manejo de enfermedades asociadas a ERC que requieran atención especializada (Estenosis de arteria renal, glomerulopatías, vasculitis, enfermedad renal hereditaria, alteraciones del metabolismo mineral óseo, entre otras).

* Recomendación (R) o punto de buena práctica clínica (BPC)

** La certeza de la evidencia solo se establece para las recomendaciones, mas no para los puntos de BPC

los establecimientos de salud de EsSalud, y 5) Uso de recursos. Luego de discutir estos criterios para cada pregunta, el GEG formuló las recomendaciones por consenso o por mayoría simple. Asimismo, el GEG formuló puntos de buenas prácticas clínicas (BPC), usualmente en base a su experiencia clínica.
Revisión por expertos externos: La presente GPC fue revisada en reuniones con médicos especialistas representantes de otras instituciones y tomadores de decisiones. Asimismo, su versión in-extenso fue enviada por vía electrónica a expertos externos para su revisión (mencionados en la sección de agradecimientos). 
El GEG tuvo en cuenta los resultados de estas revisiones para modificar las recomendaciones finales.

Aprobación de la GPC: La presente GPC fue aprobada para su uso en EsSalud, con Resolución N58-IETSI-ESSALUD-2020.

Actualización de la GPC: La presente GPC tiene una vigencia de tres años. Al acercarse al fin de este período, se procederá a realizar una RS de la literatura para su actualización, luego de la cual se decidirá si se actualiza la presente GPC o se procede a realizar una nueva versión.

\section{RECOMENDACIONES}

La presente GPC abordó 8 preguntas clínicas, con relación a cinco temas: tamizaje, diagnóstico y estadiaje; manejo farmacológico y no farmacológico; monitoreo; y criterios de referencia. En base a dichas preguntas se formularon 8 recomendaciones ( 4 recomendaciones fuertes y 4 recomendaciones condicionales), 29 puntos de buena práctica clínica, y 3 flujogramas (ver Tabla 1 y Figuras 1, 2 y 3 ).

La presente GPC se enfoca en los pacientes con ERC en estadios 1 al 3. En esta etapa de la enfermedad, el médico debe decidir cuándo realizar el tamizaje de ERC durante la atención primaria (pregunta 1), además de elegir la forma de estimar la función renal (mediante ecuaciones para calcular la tasa de filtración glomerular estimada [TFGe]) y daño renal (mediante métodos para la detección de albuminuria) (pregunta 2 y 3), definir el manejo no farmacológico y farmacológico adecuado (preguntas 4 al 7), y finalmente determinar los criterios de referencia al especialista en nefrología (pregunta 8).

A continuación, se expondrán las recomendaciones para cada pregunta clínica, así como un resumen del razonamiento seguido para llegar a cada recomendación. No se incluyó la justificación de los puntos de BPC, la cual se puede leer en el documento in-extenso.

Pregunta 1: En adultos, ¿se debería realizar el tamizaje de enfermedad renal crónica durante la atención primaria?

Evidencia: Se consideró el tamizaje dirigido a grupos de riesgo ${ }^{[1]}$. En la búsqueda sistemática no se encontró ninguna RS ni ensayo clínico aleatorizado (ECA) que hayan evaluado el efecto de realizar el tamizaje de ERC. Adicionalmente, se contó con una revisión narrativa y una GPC que proporcionaron información para la evaluación de los daños del tamizaje ${ }^{[1,9]}$

Beneficios: Si bien no se encontró estudios que hayan evaluado los beneficios de realizar tamizajes de ERC versus no realizarlos, se consideró que un tamizaje podría captar tempranamente a un importante número de pacientes con ERC en quienes se podría intervenir para evitar la progresión de la enfermedad. Para ello, se tomó en cuenta que la prevalencia de ERC en Perú se ha descrito en $20,7 \%$ para una localidad urbana de Lima y $12,9 \%$ para una localidad semiurbana de Tumbes en el año $2011^{[4]}$.
Daños: Podría presentarse estigmatización o ansiedad del paciente producto del diagnóstico, aparición de eventos adversos por el uso de procedimientos diagnósticos o tratamientos innecesarios, y el uso inadecuado de recursos en caso de falsos positivos de ERC ${ }^{[1,9]}$

Costos: Si bien el tamizaje de ERC es una intervención costosa a nivel poblacional, el GEG consideró que los costos de la hemodiálisis (calculados en 44414 soles por año), que es una de las terapias de reemplazo renal para el último estadio de la enfermedad, podrían ser mayores al costo de tamizaje y tratamiento temprano, en especial en población de riesgo. Esto está respaldado por un estudio de costo-efectividad realizado en EsSalud que aún no ha sido publicado.

Balance y fuerza: El tamizaje tiene un potencial importante para detectar pacientes con ERC y a través de un manejo adecuado, evitar su progresión a la necesidad de terapia de reemplazo renal; lo cual en opinión del GEG podría superar los daños y costos potenciales. Por ello, se emitió una recomendación a favor de realizar el tamizaje dirigido a esta población. A pesar de que la certeza de evidencia fue muy baja y no se evaluaron desenlaces importantes para los pacientes como incidencia de terapia de reemplazo renal o complicaciones de ERC, el GEG consideró que el potencial beneficio de disminuir el riesgo de requerimiento de hemodiálisis sería muy importantes en nuestro contexto. Por ello, la fuerza de la recomendación fue fuerte.

Pregunta 2: En adultos, ¿qué ecuación se debería utilizar para calcular la tasa de filtración glomerular estimada: ecuación Colaboración Epidemiológica para Enfermedad Renal Crónica (CKD-EPI) o la ecuación de Modificación de la Dieta en Enfermedad Renal con dilución isotópica con espectrometría de masa (MDRD4)?

Evidencia: Se decidió evaluar las ecuaciones MDRD4-IDMS [10] y CKD-EPI ${ }^{[11]}$ específicamente en población latinoamericana ya que el rendimiento de las ecuaciones podría variar según etnia ${ }^{[12,13]}$. En la búsqueda sistemática no se encontró ninguna RS para dicha población. Por ello, se realizó una RS de novo para población latinoamericana y se metaanalizaron los seis estudios encontrados.

Hallazgos: Para el punto de corte $<60 \mathrm{ml} / \mathrm{min} / 1,73 \mathrm{~m}^{2}$, las ecuaciones CKD-EPI creatinina y MDRD4-IDMS obtuvieron similar sensibilidad y especificidad. Sin embargo, CKD-EPI tuvo menor sesgo y mayor exactitud (P30), por lo que clasificaría mejor la severidad de la ERC.

Recomendación: Debido a las ventajas observadas con el uso de la ecuación CKD-EPI y por no involucrar mayor costo se emitió una recomendación a favor del uso de la ecuación CKD-EPI creatinina.

Fuerza: La certeza de la evidencia fue de baja a muy baja y no se contaba con información directa acerca del impacto de la capacidad diagnóstica de ambas ecuaciones en desenlaces clínicos. Por ello, la fuerza de la recomendación fue condicional. 
Tabla 2. Significado de los niveles de certeza de la evidencia y de la fuerza de la recomendación.

\section{Significado}

Certeza de la evidencia

\begin{tabular}{|c|c|}
\hline$(\bigoplus \bigoplus \bigoplus \bigoplus)$ Alta & Es muy probable que el verdadero efecto sea similar al efecto estimado. \\
\hline$(\oplus \oplus \oplus \bigcirc)$ Moderada & $\begin{array}{l}\text { Es moderadamente probable que el verdadero efecto sea similar al efecto estimado, pero es } \\
\text { posible que sea sustancialmente diferente. }\end{array}$ \\
\hline$(\bigoplus \bigoplus О \bigcirc)$ Ваја & $\begin{array}{l}\text { Nuestra confianza en el efecto es limitada. El verdadero efecto podría ser sustancialmente } \\
\text { diferente al efecto estimado. }\end{array}$ \\
\hline (๑О૦О) Muy baja & $\begin{array}{l}\text { Nuestra confianza en el efecto es pequeña. El verdadero efecto probablemente sea } \\
\text { sustancialmente diferente al efecto estimado. }\end{array}$ \\
\hline \multicolumn{2}{|l|}{ Fuerza de la recomendación } \\
\hline $\begin{array}{l}\text { Recomendación fuerte (a favor o } \\
\text { en contra) }\end{array}$ & $\begin{array}{l}\text { El GEG considera que todos o casi todos los profesionales que revisan la evidencia disponible } \\
\text { seguirían esta recomendación. En la formulación de la recomendación se usa el término "se } \\
\text { recomienda". }\end{array}$ \\
\hline $\begin{array}{l}\text { Recomendación condicional (a } \\
\text { favor o en contra) }\end{array}$ & $\begin{array}{l}\text { El GEG considera que la mayoría de los profesionales que revisan la evidencia disponible } \\
\text { seguirían esta recomendación, pero un grupo de profesionales podría optar por no } \\
\text { aplicarlas en alguna población particular, siempre que sea justificado. En la formulación de la } \\
\text { recomendación se usa el término "se sugiere". }\end{array}$ \\
\hline
\end{tabular}

Pregunta 3: En adultos, ¿qué método se debería utilizar para la detección de albuminuria: relación albuminuria-creatinuria $(\mathrm{RAC})$, relación proteinuria-creatinuria (RPC) o tiras reactivas en orina?

Evidencia: Se consideró definir y clasificar la magnitud de la albuminuria en base a los criterios propuestos por KDIGO en $2012^{[2]}$; establecer como gold standard a la albuminuria en 24 horas o a la RAC; y se tomó en cuenta que el seguro social solo cuenta con tiras reactivas para examen rutinario de orina ${ }^{[14]}$.

En la búsqueda sistemática sólo se encontró una RS que realizó metaanálisis (MA) para evaluar la capacidad diagnóstica de la RAC ${ }^{[15]}$. Se actualizó la búsqueda y MA de dicha RS con tres estudios encontrados ${ }^{[16-18]}$. En contraste, no se encontraron RS que hayan abordado la pregunta de interés para RPC ni para el tipo de tira reactiva con el que cuenta EsSalud, por lo cual solo se formularon puntos de BPC.

Hallazgos: La sensibilidad y especificidad de la RAC fue 0,87 (IC 95\%: 0,82-0,91) y 0,88 (IC 95\%: 0,85-0,91), respectivamente.

Recomendación: La RAC tendría una adecuada capacidad de identificar y, por consiguiente, tratar oportunamente a personas con ERC en estadios iniciales. En base a ello, se emitió una recomendación a favor del uso de RAC.

Fuerza: Si bien la certeza de evidencia fue baja para el uso de RAC, el GEG consideró que su uso podría devenir en beneficios y ahorros a futuro ya que permitiría detectar pacientes en estadios tempranos y que su implementación es factible. Por ello, la fuerza de la recomendación fue fuerte.
Pregunta 4: En adultos con enfermedad renal crónica en estadio 1 al 3, ¿qué tipo de dieta se debería brindar: dieta baja en proteínas (DBP), muy baja en proteínas (DMBP) o normoproteica?

\section{Pregunta 4.1: Pacientes con ERC sin diabetes mellitus}

Evidencia: En la búsqueda sistemática se encontraron tres RS de las cuales se eligió las de mejor calidad ${ }^{[19,20]}$. La población incluida en las RS fue heterogénea e incluyó participantes con ERC en estadio 3 y 4.

Beneficios: La mortalidad, incidencia de ERC estadio 5, y el cambio en la TFGe fueron similares entre aquellos que recibieron DBP y aquellos con dieta normoproteica ${ }^{[19]}$. La incidencia de ERC estadio 5 fue menor en aquellos que recibieron DMBP en comparación con aquellos que recibieron DBP o dieta normoproteica ${ }^{[19]}$, y la proteinuria fue menor con el uso de DBP o DMBP en comparación con la dieta normoproteica ${ }^{[20]}$.

Daños: El peso al final de la intervención fue menor en los pacientes que recibieron DBP ${ }^{[19]}$. El GEG consideró que esta disminución de peso sería a expensas de la disminución de masa muscular. No se han reportado la comparación de DMBP y dieta normoproteica.

Balance y fuerza para el uso de DBP: Se consideró que el beneficio en términos de disminución de proteinuria no fue clínicamente significativo, en tanto que existe un potencial daño por disminución de peso a expensas de masa muscular, y mayores costos debido a que un porcentaje de pacientes requerirán suplementos de cetoácidos y aminoácidos. Por ello, se emitió una 
recomendación en contra. Debido a que la certeza de evidencia fue muy baja, la fuerza de la recomendación fue condicional.

Balance y fuerza para el uso de DMBP: A pesar de que la DMBP podría reducir la incidencia de ERC estadio 5 , este beneficio fue observado en pacientes con estadio 4 de la ERC y el GEG consideró que este beneficio podría no estar presente en estadios tempranos. Además, podría esperarse una disminución de peso como se reportó para el uso de DBP, y devendría en costos adicionales por requerir suplementación. Por ello, se emitió una recomendación en contra. Si bien la certeza de evidencia fue baja, los posibles beneficios no superarían los potenciales daños y costos. Por ello, la fuerza de la recomendación fue fuerte.

\section{Pregunta 4.2: Pacientes con ERC y diabetes mellitus}

Evidencia: En la búsqueda sistemática se encontraron dos RS, de las cuales se eligió la de mejor calidad ${ }^{[21]}$. En la RS elegida, la población estuvo compuesta por adultos con un promedio de TFGe de $62 \mathrm{ml} / \mathrm{min} / 1,73 \mathrm{~m}^{2}$.

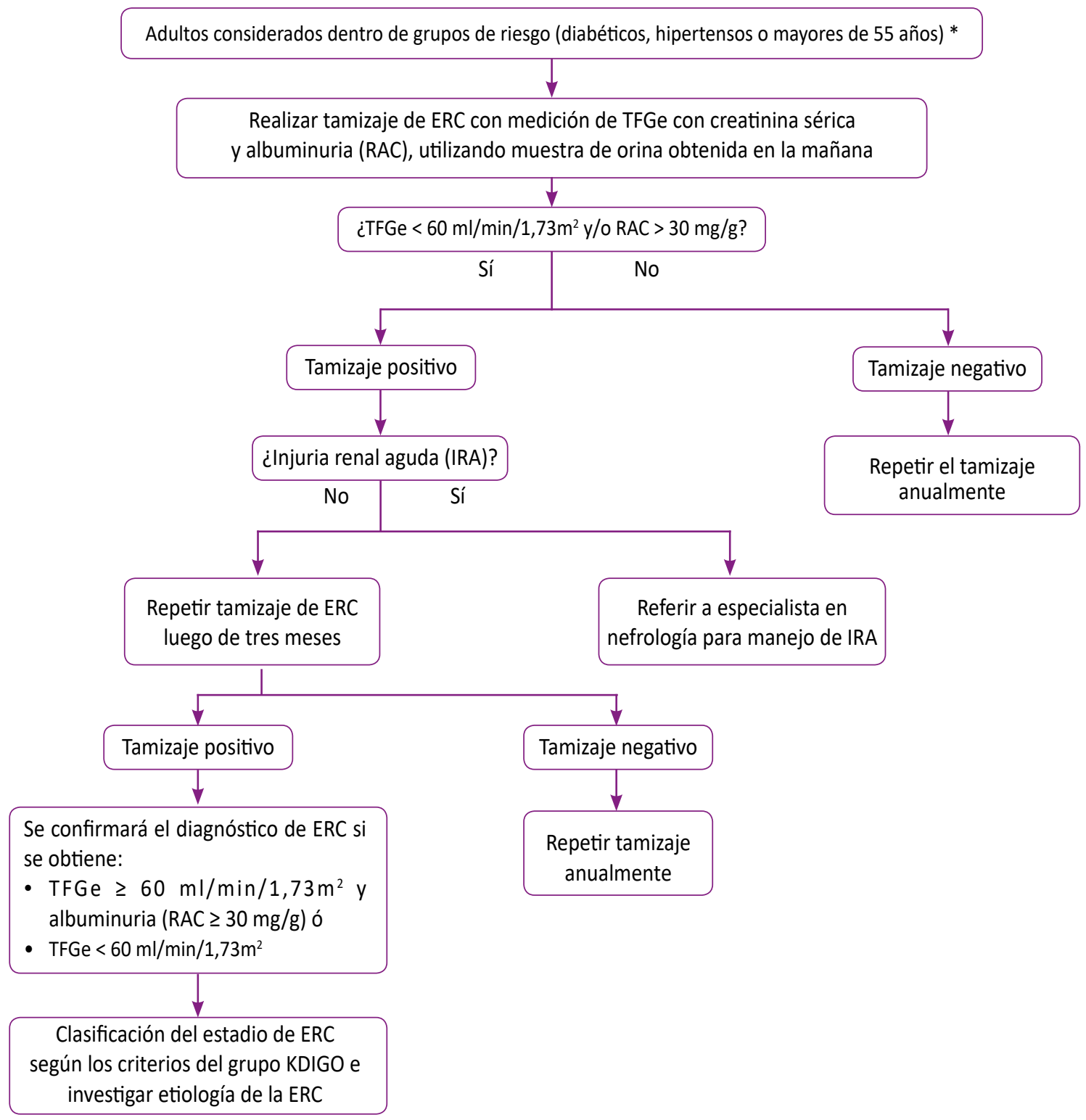

ERC: Enfermedad renal crónica; IRA: Injuria renal aguda; KDIGO: Kidney Disease Improving Global Outcomes; RAC: Relación albúminacreatinina; TFGe: Tasa de filtración glomerular estimada con creatinina sérica, calculada con la ecuación CKD-EPI creatinina.

*Considerar realizar tamizaje a personas con otros factores de riesgo para ERC como: Antecedente de IRA, enfermedades cardiovasculares, enfermedades que alteren la estructura renal o el tracto urinario, enfermedades multisistémicas con potencial daño renal, historia familiar de falla renal, presencia de hematuria, escenarios laborales con potencial daño renal (por ejemplo, exposición a pesticidas, deshidratación).

Figura 1. Flujograma de tamizaje, diagnóstico y estadiaje de pacientes con ERC. 


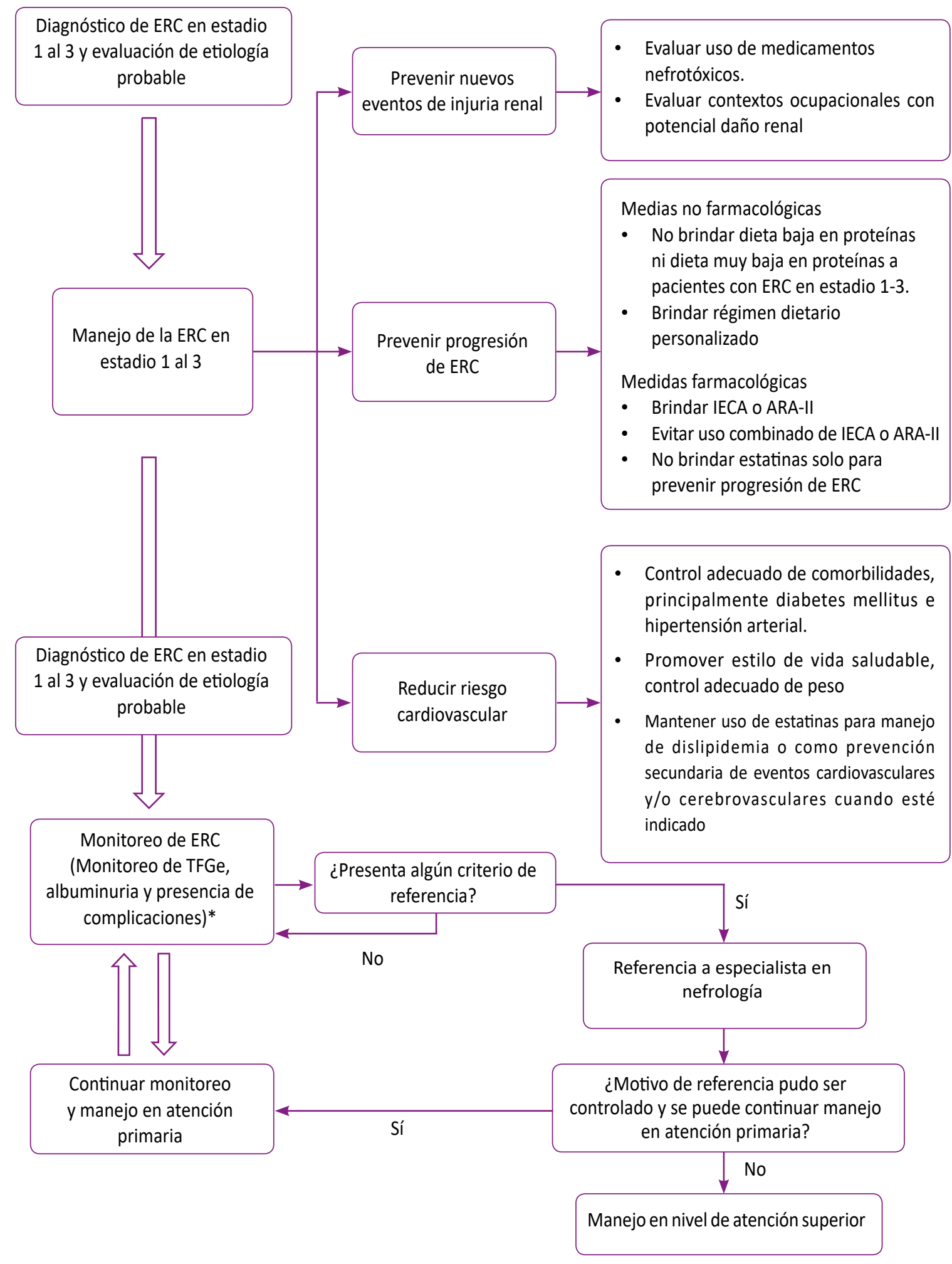

ERC: Enfermedad renal crónica; TFGe: Tasa de filtración glomerular estimada con creatinina sérica, calculada con la ecuación CKD-EPI creatinina.

*Frecuencia de monitoreo y criterios de referencia se encuentran en el flujograma sobre dicho tópico. Considerar monitorear complicaciones: hipercalemia, acidosis metabólica, hiperfosfatemia, deficiencia de vitamina D, hiperparatiroidismo secundario, anemia, o alteraciones del metabolismo mineral óseo.

Tabla 2. Flujograma de manejo no farmacológico y farmacológico de pacientes con ERC. 
Beneficios: El cambio de la TFGe fue similar entre aquellos con DBP y con dieta normoproteica, tanto en pacientes con ERC y DM tipo 1 ó $2^{[21]}$.

Daños: Se consideraron los resultados encontrados en pacientes con ERC sin DM como evidencia indirecta ${ }^{[19]}$.

Balance y fuerza: No hubo beneficio de la DBP para disminuir la progresión de ERC, por el contrario, podría causar disminución de peso a expensas de la masa muscular, e incrementaría los costos en caso de añadir suplementos nutricionales. Por ello, se emitió una recomendación en contra. Debido a que la certeza de evidencia fue muy baja, la fuerza de la recomendación fue condicional.

Pregunta 5: En adultos con enfermedad renal crónica en estadio 1 al 3 e hipertensión arterial, ¿̇se debería brindar inhibidores de la enzima convertidora de angiotensina (IECA) o antagonistas del receptor de angiotensina-II (ARA-II) para prevenir la progresión de la enfermedad renal crónica?

Evidencia: En la búsqueda sistemática se encontraron cinco RS, de las cuales se eligieron las dos de mejor calidad. Una incluyó a adultos con ERC, hipertensión arterial (HTA) y DM, la mayoría de los cuales cursaba con albuminuria ${ }^{[22]}$; mientras que la otra evaluó adultos hipertensos con ERC sin DM ${ }^{[23]}$.

Beneficios: Aquellos que utilizaron IECA o ARA-II tuvieron menor riesgo de progresión a ERC estadio $5^{[22,23]}$ y albuminuria ${ }^{[23]}$ en comparación con los que recibieron placebo o no tratamiento. Además, en pacientes con ERC, HTA y DM que cursaron con albuminuria, aquellos que utilizaron estos antihipertensivos tuvieron menor mortalidad ${ }^{[22]}$.

Daños: Aquellos que utilizaron IECA o ARA-II tuvieron mayor riesgo de hipercalemia pero similar riesgo de hipotensión, en comparación con aquellos que utilizaron placebo o no tratamiento ${ }^{[23]}$.

Balance y fuerza: Los beneficios encontrados fueron considerados estrategias críticas para disminuir el riesgo de progresión de la ERC y se consideró que los daños eran manejables. Por ello, se emitió una recomendación a favor del uso de IECA o ARA-II para evitar la progresión de la ERC. Debido a que la certeza de evidencia fue moderada, la fuerza de la recomendación fue fuerte.

Pregunta 6: En adultos con enfermedad renal crónica en estadio 1 al 3 y dislipidemia, ¿se debería brindar estatinas para prevenir la progresión de la enfermedad renal crónica?

Evidencia: En la búsqueda sistemática se encontraron seis RS, de las cuales se eligió la de mejor calidad y que proporcionara evidencia en pacientes con estadios iniciales de ERC ${ }^{[24]}$.
Adicionalmente, se tomó en cuenta una RS que proporcionó evidencia indirecta para la evaluación de los daños del uso de estatinas en diferentes tipos de patologías ${ }^{[25]}$.

Beneficios: En aquellos que usaron estatinas la albuminuria y la disminución de la TFGe anual fueron menores en comparación con quienes recibieron placebo. Sin embargo, la progresión a ERC estadio 5 fue similar ${ }^{[24]}$.

Daños: El uso de estatinas incrementó el riesgo de miositis y desarrollo de DM. Si bien la mayoría de los estudios no encontraron diferencia en la frecuencia de miopatías y rabdomiólisis, se describe que de $10 \%$ a $25 \%$ de pacientes que usa estatinas reporta problemas musculares ${ }^{[25]}$.

Balance y fuerza: Los beneficios fueron considerados pequeños ya que las estatinas no tuvieron efecto en la progresión a ERC estadio 5; y los hallazgos en albuminuria y disminución de la TFGe no serían clínicamente importantes. Los daños fueron considerados pequeños, pero podrían tener mayor impacto en grupos vulnerables. Por ello, se emitió una recomendación en contra de brindar estatinas para prevenir la progresión de ERC. Puesto que la certeza de evidencia fue baja, la fuerza de la recomendación fue condicional.

Pregunta 7: En adultos con enfermedad renal crónica en estadio 1 al 3, ¿cuál debe ser la frecuencia de monitoreo de la tasa de filtración glomerular estimada (TFGe) y albuminuria para evaluar la progresión y el estadio de la enfermedad renal crónica?

Evidencia: Se consideró que la albuminuria y TFGe son los parámetros más utilizados para realizar el monitoreo de la ERC ${ }^{[6,26]}$. En la búsqueda sistemática no se encontraron RS que aborden la pregunta. Sin embargo, se encontraron cinco RS que proporcionaban información respecto a las frecuencias en los que evaluaba la albuminuria y TFGe en estudios previos ${ }^{[27-31]}$. Por tal motivo, se optó por emitir puntos de BPC considerando las frecuencias más usadas y la factibilidad en nuestro contexto (Tabla 1).

Pregunta 8: En adultos con enfermedad renal crónica en estadio 1 al 3, ¿cuáles son los criterios de referencia al especialista en nefrología?

Evidencia: No se encontraron RS que aborden la pregunta. Se consideró que diversas GPC basan los criterios de referencia en consensos de expertos y se decidió evaluar dicha información. Para tal fin, se tenía conocimiento de dos revisiones narrativas ${ }^{[6,26]}$ que resumían las recomendaciones de GPC para el monitoreo de ERC. Se tomaron en cuenta dichas revisiones narrativas para formular puntos de BPC (Tabla 1).

Contribuciones de autoría: Todos los autores participaron en la realización de la guía. SGL, GDM, LCA y VEFR se encargaron de las 


\begin{tabular}{|c|c|c|c|c|}
\hline \multicolumn{5}{|c|}{ Monitoreo y referencia a especialista en nefrología de pacientes con ERC } \\
\hline & \multirow[b]{2}{*}{ Estadio } & \multicolumn{3}{|c|}{ Categorías de albuminuria (mg/g) } \\
\hline & & $\begin{array}{l}\text { A1 }(<30) \text { : Normal o } \\
\text { aumento leve }\end{array}$ & $\begin{array}{c}\text { A2 (30-300): Aumento } \\
\text { moderado }\end{array}$ & $\begin{array}{c}\text { A3 (>300): Aumento } \\
\text { severo }\end{array}$ \\
\hline \multirow{6}{*}{$\begin{array}{l}\text { Categorías de TFGe } \\
\left(\mathrm{ml} / \mathrm{min} / 1,73 \mathrm{~m}^{2}\right)\end{array}$} & G1 ( $\geq 90)$ : Normal o alto & \multirow{2}{*}{ No ERC } & $\begin{array}{l}\text { Monitorear* } \\
\text { ( } \pm 1 \text { vez al año) }\end{array}$ & $\begin{array}{c}\text { Monitorear }{ }^{\dagger} \\
( \pm 2 \text { veces al año) }\end{array}$ \\
\hline & G2 (60-89): Reducción leve & & $\begin{array}{l}\text { Monitorear* } \\
\text { ( } \pm 1 \text { vez al año) }\end{array}$ & $\begin{array}{c}\text { Monitorear }{ }^{\dagger} \\
( \pm 2 \text { veces al año) }\end{array}$ \\
\hline & $\begin{array}{l}\text { G3a (45-59): Reducción } \\
\text { leve-moderada }\end{array}$ & $\begin{array}{c}\text { Monitorear* } \\
\text { ( } \pm 2 \text { veces al año) }\end{array}$ & $\begin{array}{c}\text { Monitorear† } \\
( \pm 2 \text { veces al año) }\end{array}$ & $\begin{array}{c}\text { Monitorear }{ }^{\dagger} \\
\text { ( } \pm 2 \text { veces al año) }\end{array}$ \\
\hline & $\begin{array}{l}\text { G3b (30-44): Reducción } \\
\text { moderada-severa }\end{array}$ & $\begin{array}{c}\text { Monitorear }{ }^{\dagger} \\
( \pm 2 \text { veces al año) }\end{array}$ & $\begin{array}{c}\text { Monitorear† } \\
\text { ( } \pm 2 \text { veces al año) }\end{array}$ & Referirł \\
\hline & $\begin{array}{l}\text { G4 (15-29): Reducción } \\
\text { severa }\end{array}$ & Referir $\ddagger$ & Referirł & Referir $\ddagger$ \\
\hline & G5 (<15): Falla renal & Referir $\ddagger$ & Referir $\ddagger$ & Referir $\ddagger$ \\
\hline
\end{tabular}

TFGe: Tasa de filtración glomerular estimada, calculada mediante la ecuación CKD-EPI creatinina [Ver pregunta $\mathrm{N}^{\circ} 2$ ], Albuminuria: Excreción urinaria de albúmina obtenida mediante relación albuminuria-creatinuria (RAC) [ver pregunta $\mathrm{N}^{\circ} 3$ ]

* Realizar el monitoreo y manejo en atención primaria

† Realizar el monitoreo y manejo en atención primaria. Referir al especialista en nefrología cuando presenten alguno de los criterios de referencia [ver pregunta $\mathrm{N}^{\circ} 8$ ]

‡ Referir al especialista en nefrología

Nota: Sobre la frecuencia de monitoreo, queda a criterio clínico realizar un menor o mayor número de controles de TFGe y albuminuria teniendo en cuenta factores de riesgo de progresión del paciente (hipertensión arterial o diabetes mellitus con mal control, cambio en el rango de albuminuria, disminución rápida de la TFGe o nivel de TFGe).

Figura 3. Flujograma de monitoreo y referencia de pacientes con ERC

búsquedas sistemáticas, la evaluación de calidad de los estudios y la evaluación de la certeza de la evidencia para cada pregunta. $A B C, S G L$ y ATR redactaron la primera versión del artículo. Todos los autores participaron en la discusión de los estudios encontrados y la formulación de las recomendaciones y puntos de buenas prácticas clínicas. Todos los autores revisaron y aprobaron la versión final del manuscrito; y asumen la responsabilidad por el contenido del artículo.

Potenciales conflictos de intereses: Los responsables de la elaboración del presente documento declaran no tener ningún conflicto de interés financiero y no financiero, con relación a los temas descritos en el presente documento.

Fuentes de financiamiento: Este documento técnico ha sido financiado por el Instituto de Evaluación de Tecnologías en Salud e Investigación (IETSI), EsSalud, de Perú.

Material suplementario: Disponible en la versión electrónica de Act Med Peru.

\section{ORCID}

Jessica Bravo-Zúñiga, http://orcid.org/0000-0003-2528-6271

Juana Hinostoza Sayas, https://orcid.org/0000-0002-6852-3217

Sergio Goicochea-Lugo, https://orcid.org/0000-0002-0487-5547

Gandy Dolores-Maldonado, https://orcid.org/0000-0002-7146-7885

Ana Brañez-Condorena, https://orcid.org/0000-0001-5518-3025

Alvaro Taype-Rondan, https://orcid.org/0000-0001-8758-0463

Carlos Yanar Pereda Vejarano, https://orcid.org/0000-0003-2972-7740

Carola Medina Sal y Rosas, https://orcid.org/0000-0001-8269-3231

Renzo P. Valdivia-Vega, https://orcid.org/0000-0002-8718-4361

Fernando Mesías Gonzáles Haro, https://orcid.org/0000-00023299-7250

Luzmila Livaque Gaona, https://orcid.org/0000-0002-1540-3529 Victor Manuel Conetero Muro, https://orcid.org/0000-00029730-5067

Gabriela Ayllón Guerrero, https://orcid.org/0000-0003-1277-9261 Nelly Patricia Huancco Cáceres, https://orcid.org/0000-00020205-8797

César Antonio Loza Munarriz, https://orcid.org/0000-00034545-9969 
Percy Herrera-Añazco, https://orcid.org/0000-0003-0282-6634 Virgilio E. Failoc Rojas, https://orcid.org/0000-0003-2992-9342 Lourdes Carrera-Acosta, https://orcid.org/0000-0002-6852-2601 Raúl Timaná Ruiz, https://orcid.org/0000-0003-0693-3445 Héctor Garavito-Farro, https://orcid.org/0000-0002-9590-9027

\section{REFERENCIAS BIBLIOGRÁFICAS}

1. Romagnani P, Remuzzi G, Glassock R, Levin A, Jager KJ, Tonelli M, et al. Chronic kidney disease. Nat Rev Dis Primers. 2017;3:17088. doi: $10.1038 /$ nrdp.2017.88

2. Kidney Disease: Improving Global Outcomes (KDIGO) CKD Work Group. KDIGO 2012 Clinical Practice Guideline for the Evaluation and Management of Chronic Kidney Disease. Kidney inter. 2013;3 Suppl.:1-150.

3. Xie Y, Bowe B, Mokdad AH, Xian H, Yan Y, Li T, et al. Analysis of the Global Burden of Disease study highlights the global, regional, and national trends of chronic kidney disease epidemiology from 1990 to 2016. Kidney Int. 2018;94(3):567-81. doi: 10.1016/j. kint.2018.04.011.

4. Francis ER, Kuo C-C, Bernabe-Ortiz A, Nessel L, Gilman RH, Checkley $W$, et al. Burden of chronic kidney disease in resource-limited settings from Peru: a population-based study. BMC Nephrol. 2015;16(1):114. doi: 10.1186/s12882-015-0104-7.

5. Bravo-Zuniga J, Galvez-Inga J, Carrillo-Onofre P, Chavez-Gomez R, Castro-Monteverde P. Early detection of chronic renal disease: coordinated work between primary and specialized care in an ambulatory renal network of Peru. J Bras Nefrol. 2019;41(2):17684. doi: 10.1590/2175-8239-jbn-2018-0101.

6. Chen TK, Knicely DH, Grams ME. Chronic Kidney Disease Diagnosis and Management: A Review. JAMA. 2019;322(13):1294-304. doi: 10.1001/jama.2019.14745.

7. Balshem $H$, Helfand $M$, Schünemann HJ, Oxman AD, Kunz R, Brozek J, et al. GRADE guidelines: 3 . Rating the quality of evidence. J Clin Epidemiol. 2011;64(4):401-6. doi: 10.1016/j.jclinepi.2010.07.015.

8. Andrews J, Guyatt G, Oxman AD, Alderson P, Dahm P, Falck-Ytter Y, et al. GRADE guidelines: 14 . Going from evidence to recommendations: the significance and presentation of recommendations. J Clin Epidemiol. 2013;66(7):719-25. doi: 10.1016/j.jclinepi.2012.03.013.

9. Qaseem A, Hopkins RH, Sweet DE, Starkey M, Shekelle P. Screening, monitoring, and treatment of stage 1 to 3 chronic kidney disease: $A$ clinical practice guideline from the American College of Physicians. Ann Intern Med. 2013;159(12):835-47. doi: 10.7326/0003-4819159-12-201312170-00726.

10. Levey AS, Coresh J, Greene T, Marsh J, Stevens LA, Kusek JW, et al. Expressing the Modification of Diet in Renal Disease Study equation for estimating glomerular filtration rate with standardized serum creatinine values. Clin Chem. 2007;53(4):766-72. doi: 10.1373/ clinchem.2006.077180.

11. Levey AS, Stevens LA, Schmid CH, Zhang YL, Castro AF, Feldman HI, et al. A new equation to estimate glomerular filtration rate. Ann Intern Med. 2009;150(9):604-12. doi: 10.7326/0003-4819-150-9200905050-00006.

12. Teo BW, Xu H, Wang D, Li J, Sinha AK, Shuter B, et al. GFR estimating equations in a multiethnic Asian population. Am J Kidney Dis. 2011;58(1):56-63. doi: 10.1053/j.ajkd.2011.02.393.

13. Omuse G, Maina D, Mwangi J, Wambua C, Kanyua A, Kagotho E, et al. Comparison of equations for estimating glomerular filtration rate in screening for chronic kidney disease in asymptomatic black
Africans: a cross sectional study. BMC Nephrol. 2017;18(1):369. doi: 10.1186/s12882-017-0788-y.

14. Instituto de Evaluación de Tecnologías en Salud e Investigación. Petitorio Nacional de Patología Clínica y Anatomía Patológica [Internet]. Lima, Peru: EsSalud [citado el 15 de setiembre de 2020]. Disponible en: http://www.essalud.gob.pe/ietsi/PETITORIO_ DE_PATOLOGIA_CLINICA_Y_ANATOMIA_PATOLOGICA/

15. Wu H-Y, Peng Y-S, Chiang C-K, Huang J-W, Hung K-Y, Wu K-D, et al. Diagnostic performance of random urine samples using albumin concentration vs ratio of albumin to creatinine for microalbuminuria screening in patients with diabetes mellitus: a systematic review and meta-analysis. JAMA Intern Med. 2014;174(7):1108-15. doi: 10.1001/jamainternmed.2014.1363.

16. McTaggart MP, Price CP, Pinnock RG, Stevens PE, Newall RG, Lamb EJ. The diagnostic accuracy of a urine albumin-creatinine ratio point-of-care test for detection of albuminuria in primary care. Am J Kidney Dis. 2012;60(5):787-94. doi: 10.1053/j.ajkd.2012.05.009.

17. Pathania M, Rathaur VK, Yadav N, Jayara A, Chaturvedi A. Quantitative Micro-albuminuria Assessment from 'Random Voided Urinary Albumin: Creatinine Ratio'Versus '24 hours Urinary Albumin Concentration'for Screening of Diabetic Nephropathy. J Clin Diagn Res. 2013;7(12):2828-31. doi: 10.7860/JCDR/2013/6589.3768.

18. Hasanato RM. Diagnostic efficacy of random albumin creatinine ratio for detection of micro and macro-albuminuria in type 2 diabetes mellitus. Saudi Med J. 2016;37(3):268-73. doi: 10.15537/ smj.2016.3.13567.

19. Hahn D, Hodson EM, Fouque D. Low protein diets for non-diabetic adults with chronic kidney disease. Cochrane Database Syst Rev. 2018;10:CD001892. doi: 10.1002/14651858.CD001892.pub4.

20. Yan B, Su X, Xu B, Qiao X, Wang L. Effect of diet protein restriction on progression of chronic kidney disease: A systematic review and meta-analysis. PLoS One. 2018;13(11):e0206134. doi: 10.1371/ journal.pone.0206134.

21. Rughooputh MS, Zeng R, Yao Y. Protein Diet Restriction Slows Chronic Kidney Disease Progression in Non-Diabetic and in Type 1 Diabetic Patients, but Not in Type 2 Diabetic Patients: A Meta-Analysis of Randomized Controlled Trials Using Glomerular Filtration Rate as a Surrogate. PLoS One. 2015;10(12):e0145505. doi: 10.1371/journal.pone.0145505.

22. Fink HA, Ishani A, Taylor BC, Greer NL, MacDonald R, Rossini D, et al. Screening for, monitoring, and treatment of chronic kidney disease stages 1 to 3: a systematic review for the US Preventive Services Task Force and for an American College of Physicians Clinical Practice Guideline. Ann Intern Med. 2012;156(8):570-81. doi: 10.7326/0003-4819-156-8-201204170-00008.

23. Mishima E, Haruna $Y$, Arima H. Renin-angiotensin system inhibitors in hypertensive adults with non-diabetic CKD with or without proteinuria: a systematic review and meta-analysis of randomized trials. Hypertens Res. 2019;42(4):469-82. doi: 10.1038/s41440018-0116-3.

24. Su X, Zhang L, Lv J, Wang J, Hou W, Xie X, et al. Effect of statins on kidney disease outcomes: a systematic review and metaanalysis. Am J Kidney Dis. 2016;67(6):881-92. doi: 10.1053/j. ajkd.2016.01.016

25. He Y, Li X, Gasevic D, Brunt E, McLachlan F, Millenson M, et al. Statins and Multiple Noncardiovascular Outcomes: Umbrella Review of Meta-analyses of Observational Studies and Randomized Controlled Trials. Ann Intern Med. 2018;169(8):543-53. doi: 10.7326/m18-0808.

26. Weckmann GFC, Stracke S, Haase A, Spallek J, Ludwig F, Angelow $A$, et al. Diagnosis and management of non-dialysis chronic kidney disease in ambulatory care: a systematic review of clinical practice 
guidelines. BMC Nephrol. 2018;19(1):258. doi: 10.1186/s12882018-1048-5.

27. Naimark DM, Grams ME, Matsushita K, Black C, Drion I, Fox CS, et al. Past Decline Versus Current eGFR and Subsequent Mortality Risk. J Am Soc Nephrol. 2016;27(8):2456-66. doi: 10.1681/ asn.2015060688.

28. Kovesdy CP, Coresh J, Ballew SH, Woodward M, Levin A, Naimark DM, et al. Past Decline Versus Current eGFR and Subsequent ESRD Risk. J Am Soc Nephrol. 2016;27(8):2447-55. doi: 10.1681/ asn.2015060687.

29. Badve SV, Palmer SC, Hawley CM, Pascoe EM, Strippoli GF, Johnson DW. Glomerular filtration rate decline as a surrogate end point in kidney disease progression trials. Nephrol Dial Transplant. 2016;31(9):1425-36. doi: 10.1093/ndt/gfv269.

30. Coresh J, Heerspink HJL, Sang Y, Matsushita K, Arnlov J, Astor BC, et al. Change in albuminuria and subsequent risk of end-stage kidney disease: an individual participant-level consortium meta-analysis of observational studies. Lancet Diabetes Endocrinol. 2019;7(2):11527. doi: 10.1016/s2213-8587(18)30313-9.

31. Heerspink HJL, Greene T, Tighiouart H, Gansevoort RT, Coresh J, Simon AL, et al. Change in albuminuria as a surrogate endpoint for progression of kidney disease: a meta-analysis of treatment effects in randomised clinical trials. Lancet Diabetes Endocrinol. 2019;7(2):128-39. doi: 10.1016/s2213-8587(18)30314-0. 